\title{
КОМПЬЮТЕРНОЕ МОДЕЛИРОВАНИЕ ВЗАИМОДЕЙСТВИЯ ПРЕДЕЛЬНО КОРОТКИХ ОПТИЧЕСКИХ ИМПУЛЬСОВ В СИЛИЦЕНЕ
}

\author{
Конобеева Наталия Николаевна \\ Кандидат физико-математических наук, доцент кафедры информационных систем \\ и компьютерного моделирования, \\ Волгоградский государственный университет \\ yana_nn@inbox.ru, infomod@volsu.ru \\ просп. Университетский, 100, 400062 г. Волгоград, Российская Федерация
}

Аннотация. Рассмотрена задача о моделировании распространения и взаимодействия двух предельно коротких оптических импульсов в силицене. Электроны описывались на основании длинноволнового эффективного гамильтониана в случае низких температур. Выявлена зависимость от скорости и амплитуды предельно короткого импульса.

Ключевые слова: компьютерное моделирование, силицен, предельно короткие оптические импульсы, столкновение, уравнения Максвелла.

\section{Введение}

Экспериментальные работы по получению двумерных кристаллов, которые продемонстрировали давно ожидаемые особенности в поведении низкоразмерных систем, вызвали огромной всплеск научно-исследовательской деятельности в этом направлении. Одним из примеров подобной структуры является графен, обладающий уникальными электрофизическими свойствами $[10 ; 11]$. Вместе с тем отсутствие запрещенной зоны в чистом графене вынуждает исследователей изучать его различные модификации. Хотя в образцах графена на подложке никеля и удалось существенно увеличить величину спин-орбитального взаимодействия [5], это было признано недостаточным, и внимание исследователей переключилось на другие графеноподобные двумерные кристаллы с более сильным спин-орбитальным взаимодействием. Таким материалом, например, является силицен - монослой атомов кремния в гексагональной решетке [6;9;12]. Одним из интересных предсказаний для силицена является возникновение запрещенной зоны, что привело бы к переходу между топологическим изолятором и зонным изолятором. К тому же нельзя оставить без внимания тот 
факт, что кремний все еще остается базовым элементом для устройств современной микроэлектроники.

Опираясь на вышеизложенные обстоятельства, представляется достаточно важным и актуальным исследовать взаимодействие предельно коротких оптических импульсов в силицене на основе построенной компьютерной модели.

\section{1. Математическая модель задачи}

Используем математическую модель длинноволнового приближения, в которой гамильтониан для силицена можно записать в виде [7; 8]:

$$
H=v\left(\xi k_{x} \sigma_{x}+k_{y} \sigma_{y}\right)-0,5 \xi \Delta_{S O} \tau_{z} \sigma_{z}+0,5 \Delta_{z} \sigma_{z}
$$

где $\xi- \pm$ знак долины для двух дираковских точек; $v$ - скорость дираковских электронов; $\boldsymbol{p}=\left(k_{x}, k_{y}\right)-$ квазиимпульс электронов; $\Delta_{\mathrm{SO}}-$ величина спин-орбитальной щели для силицена; $\Delta_{\mathrm{z}}$ - потенциал на одном узле решетки, причем $\Delta_{\mathrm{z}}=E_{z} d, E_{z}$ - электрическое поле; $d$ - расстояние между двумя подрешеточными плоскостями; $\sigma_{\mathrm{i}}, \tau_{\mathrm{i}}-$ матрицы Паули.

В матричной форме гамильтониан имеет вид:

$$
\hat{H}_{\sigma \xi}=\left(\begin{array}{cc}
-0.5 \sigma \xi \Delta_{S O}+0.5 \Delta_{z} & v\left(\xi k_{x}-i k_{y}\right) \\
v\left(\xi k_{x}+i k_{y}\right) & 0.5 \sigma \xi \Delta_{S O}-0.5 \Delta_{z}
\end{array}\right),
$$

где $\sigma-$ спин электрона (спин «вверх» и «вниз»).

Собственные числа можно получить из выражения (2):

$$
\varepsilon_{\sigma \xi}= \pm \sqrt{v^{2} k^{2}+\frac{1}{4}\left(\Delta_{z}-\sigma \xi \Delta_{S O}\right)^{2}}
$$

Как следует из правил квантовой механики в присутствии внешнего электрического поля, которое для определенности направлено вдоль оси $z$ и будет далее рассматриваться в калибровке: $\boldsymbol{E}=-\partial \boldsymbol{A} / \mathrm{c} \partial t$, необходимо заменить импульс на обобщенный импульс: $p \rightarrow p-e A / c(\mathrm{e}-$ заряд электрона). В этом случае гамильтониан (1) можно переписать как:

$$
H=\sum_{p \sigma} \varepsilon\left(p-\frac{e}{c} A(t)\right) a_{p \sigma}^{+} a_{p \sigma},
$$

где $a_{p \sigma}^{+}, a_{p \sigma}$-операторы рождения, уничтожения электронов с квазиимпульсом $p$ и спином $\sigma$.

Уравнения Максвелла с учетом калибровки можно записать в следующем виде [4]:

$$
\frac{\partial^{2} \boldsymbol{A}}{\partial x^{2}}-\frac{1}{c^{2}} \frac{\partial^{2} \boldsymbol{A}}{\partial t^{2}}+\frac{4 \pi}{c} \boldsymbol{j}=0
$$

Вектор-потенциал выбирается в виде $\boldsymbol{A}=(0, A(x, t), 0)$.

Запишем стандартное выражение для плотности тока:

$$
j=e \sum_{p} v_{y}\left(p-\frac{e}{c} A(x, t)\right)\left\langle a_{p}^{+} a_{p}\right\rangle
$$


где $v_{y}(p)=\frac{\partial \varepsilon\left(p_{x}, p_{y}\right)}{\partial p_{y}}$, скобки означают усреднение с неравновесной матрицей плотности $\rho(t):\langle B\rangle=\operatorname{Sp}(B(0) \rho(t))$.

Далее рассмотрим случай низких температур, когда в сумму (4) дает вклад лишь небольшая область в импульсном пространстве вблизи уровня Ферми. Соответственно этому формула (5) перепишется в виде:

$$
j=e \int_{-\Delta}^{\Delta} \int_{-\Delta}^{\Delta} d p_{x} d p_{y} v_{y}\left(p-\frac{e}{c} A(x, t)\right)
$$

Область интегрирования по импульсам в (6) определим из условия равенства числа частиц:

$$
\int_{-\Delta}^{\Delta} \int_{-\Delta}^{\Delta} d p_{x} d p_{y}=\int_{Z B} d p_{x} d p_{y}\left\langle a_{p x, p y}^{+} a_{p x, p y}\right\rangle
$$

интегрирование справа ведется по первой зоне Бриллюэна [3].

При этом уравнение на распространение предельно короткого импульса можно записать как:

$$
\frac{\partial^{2} A}{\partial x^{2}}-\frac{1}{c^{2}} \frac{\partial^{2} A}{\partial t^{2}}+\frac{4 \pi}{c} \Phi(A)=0
$$

а $\Phi(A)$ определяется интегрированием в (6).

Исследуемое уравнение (7) решалось численно при помощи прямой разностной схемы типа крест [1]. Начальный профиль каждого импульса выбирался в гауссовой форме, а именно, в виде предельно короткого импульса, состоящего из одного колебания, что соответственно задает следующее условие на $A$ :

$$
\begin{aligned}
& A_{i}(x, t)=B_{i} \exp \left(-\left(x-v_{i} t\right)^{2} / \gamma_{i}\right) \\
& \gamma_{i}=\left(1-v_{i}^{2}\right)^{1 / 2}, i=1,2
\end{aligned}
$$

где $B_{i}$ - амплитуда, а $v_{i}$ - начальная скорость $i$-го предельно короткого импульса на входе в образец.

Амплитуда импульсов и их скорости выбирались одинаковыми. Значения энергетических параметров выражались в единицах $\Delta$.

\section{2. Результаты компьютерного моделирования}

Возникающая эволюция электромагнитного поля при его распространении по образцу представлена на рисунке 1.

Из рисунка 1 видно, что в процессе эволюции сталкивающиеся импульсы распадаются на несколько импульсов. Затем происходит удаление предельно коротких импульсов друг от друга с сохранением амплитуды.

Влияние начальной амплитуды импульса представлено на рисунке 2.

В случае столкновения импульсов с разными амплитудами наблюдается рассеивание уединенного импульса с меньшей амплитудой на импульсе с большей амплитудой, вследствие чего импульс с меньшей амплитудой исчезает. 

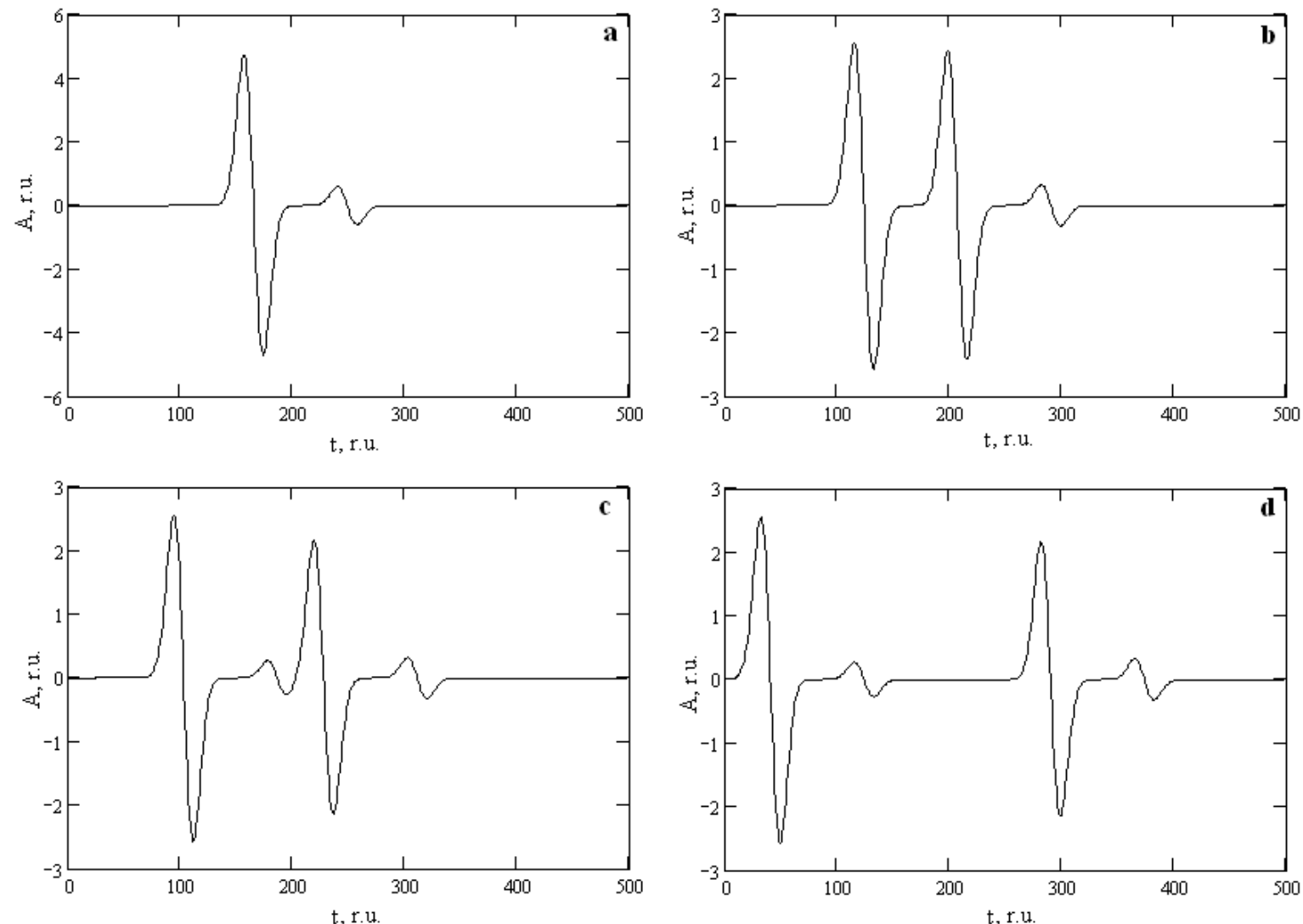

Рис. 1. Зависимость вектор-потенциала (в единицах $\left.10^{6} \mathrm{~B} / \mathrm{M}\right)$ от времени (в единицах $10^{-13}$ с) для разных точек пространства: a) $\left.\left.x=3,0 \cdot 10^{-8} \mathrm{M} ; b\right) x=5 \cdot 10^{-6} \mathrm{M} ; c\right) x=7,5 \cdot 10^{-6} \mathrm{M}$; $d$ ) $x=1,5 \cdot 10^{-5} \mathrm{M}$

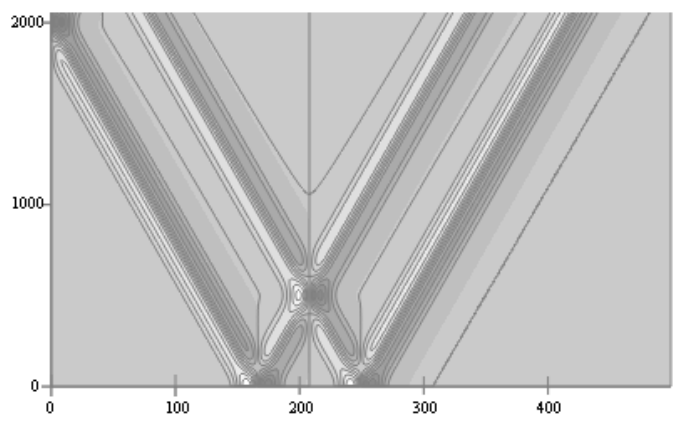

(a)

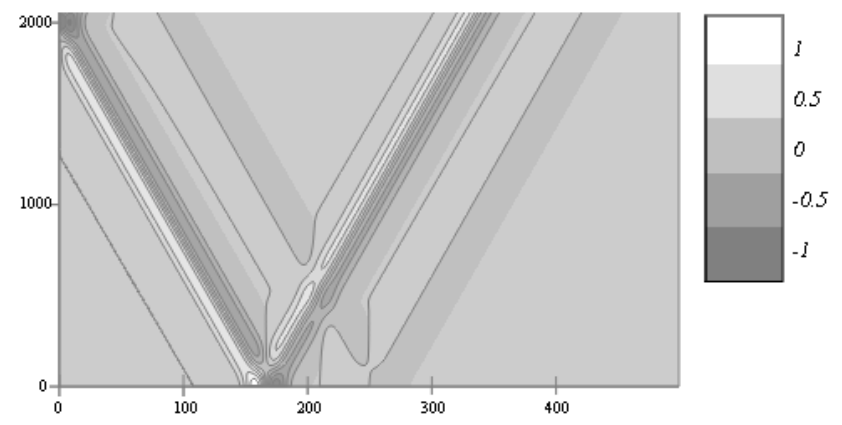

(b)

Рис. 2. Картина столкновения двух импульсов. Яркость соответствует величине электрического поля импульса в относительных единицах. Время по вертикальной оси (в единицах $\left.10^{-13} \mathrm{c}\right)$, по горизонтальной оси координата. $v_{1}=-v_{2}=0,85$ c: $\left.a\right) B_{1}=B_{2}=0,5$ r.u.; $\left.b\right) B_{1}=0,5$ r.u., $B_{2}=4,0$ r.u.

Это можно связать, с тем, что происходит нелинейное взаимодействие, в результате которого происходит перераспределение энергии импульсов, что, в свою очередь, приводит к появлению за импульсом с большей амплитудой более длинного «хвоста». Данное взаимодействие, несомненно, может оказаться полезным в устройствах управления светом при помощи света и может служить, например, базой, для аналогового компаратора амплитуд импульсов. 


\section{КОМПЬЮТЕРНОЕ МОДЕЛИРОВАНИЕ}

Двумерная картина столкновения двух импульсов разной амплитуды представлена на рисунке 3 .

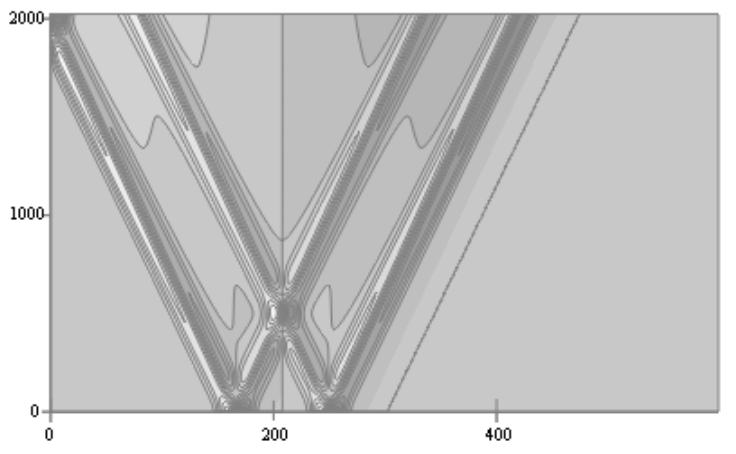

(a)

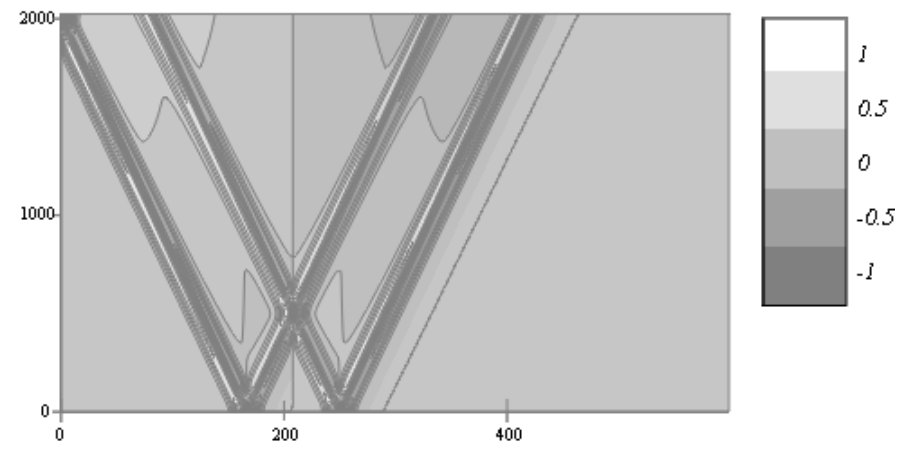

(b)

Рис. 3. Картина столкновения двух импульсов. Яркость соответствует величине электрического поля импульса в относительных единицах. Время по вертикальной оси (в единицах $\left.10^{-13} \mathrm{c}\right)$, по горизонтальной оси координата. $B_{1}=B_{2}=0,5$ r.u.: $\left.\left.a\right) v_{1}=-v_{2}=0,85 \mathrm{c} ; b\right) v_{1}=-v_{2}=0,99 \mathrm{c}$

Подобное поведение наблюдалось и в других работах авторов [2] и имеет достаточно простую физическую интерпретацию. С увеличением скорости уменьшается как величина пространственной локализации уединенного импульса, так и время, за которое один импульс «проходит» через другой. Все это приводит к тому, что эффекты, связанные с нелинейным взаимодействием импульсов, не успевают развиться, и столкновение происходит «упругим» образом (то есть без образования за импульсами хвостов).

\section{СПИСОК ЛИТЕРАТУРЫ}

1. Бахвалов, Н. С. Численные методы (анализ, алгебра, обыкновенные дифференциальные уравнения) / Н. С. Бахвалов. - М. : Наука, 1975. - 632 с.

2. Галкина, Е. Н. Распространение предельно коротких импульсов в углеродных нанотрубках в присутствии магнитного поля / Е. Н. Галкина, М. Б. Белоненко // Известия РАН. Серия физическая. - 2011. Т. 75, вып. 12. - С. 1727-1729.

3. Конобеева, Н. Н. Динамика предельно коротких электромагнитных импульсов в силиценовых волноводах / Н. Н. Конобеева, М. Б. Белоненко // ПЖТФ. - 2013. - Т. 39, вып. 12. - С. 87-94.

4. Ландау, Л. Д. Теоретическая физика. Т. ІІ. Теория поля / Л. Д. Ландау, Е. М. Лифшиц. - М. : Наука, 1988. $-512 \mathrm{c.}$

5. Янюшкина, Н. Н. Влияние спин-орбитального взаимодействия на динамику предельно коротких импульсов в графеновых системах / Н. Н. Янюшкина, М. Б. Белоненко, Н. Г. Лебедев // Оптика и спектроскопия. - 2012. - Т. 112 (3). - С. 498-501.

6. Aufray, B. Graphene-like silicon nanoribbons on Ag (110): A possible formation of silicene / B. Aufray, A. Kara, S. Vizzini, H. Oughaddou, C. Leandri, B. Ealet, G. Le Lay // Appl. Phys. Lett. - 2010. - Vol. 96. P. 183102.

7. Ezawa, M. Topological insulator and helical zero mode in silicone under inhomogeneous electric field / M. Ezawa // New J. Phys. - 2012. - Vol. 14. - P. 033003.

8. Ezawa, M. Valley-polarized metals and quantum anomalous Hall effect in silicone / M. Ezawa // Phys. Rev. Lett. - 2012. - Vol. 109. - P. 055502.

9. Lalmi, B. Epitaxial growth of a silicene sheet / B. Lalmi, H. Oughaddou, H. Enriquez, A. Kara, S. Vizzini, B. Ealet, B. Aufray // Appl. Phys. Lett. - 2010. - Vol. 97. - P. 223109.

10. Novoselov, K. S. Electric field effect in atomically thin carbon films / K. S. Novoselov, A. K. Geim, 
S. V. Morozov, D. Jiang, Y. Zhang, S. V. Dubonos, I. V. Grigorieva, A. A. Firsov // Science. - 2004. Vol. 306. - P. 666-669.

11. Novoselov K. S. Two-dimensional atomic crystals / K. S. Novoselov, D. Jiang, F. Schedin, T. J. Booth, V. V. Khotkevich, S. V. Morozov, A. K. Geim // Proc. NatlAcad. Sci. USA. - 2005. - Vol. 102 (No. 30). P.10451.

12. Padova, P. Evidence of graphene-like electronic signature in silicone nanoribbons / P. Padova, C. Quaresima, C. Ottaviani, P. Sheverdyaeva, P. Moras, C. Carbone, D. Topwal, B. Olivieri, A. Kara, H. Oughaddou, B. Aufray, G. Lay // Appl. Phys. Lett. - 2010. - Vol. 96. - P. 261905.

\section{REFERENCES}

1. Bakhvalov N.S. Chislennye metody (analiz, algebra, obyknovennye differentsialnye uravneniya) [Numerical Methods (Analysis, Algebra, Differential Equations)]. Moscow, Nauka Publ.,1975. 632 p.

2. Galkina E.N., Belonenko M.B. Rasprostranenie predelno korotkikh impulsov v uglerodnykh nanotrubkakh v prisutstvii magnitnogo polya [Propagation of Ultra-Short Pulses in Carbon Nanotubes in the Presence of Magnetic Field]. Izvestiya RAN. Seriya fizicheskaya [Bulletin of RAS. Physics], 2011, vol. 75 (12), pp. 1727-1729.

3. Konobeeva N.N., Belonenko M.B. Dinamika predelno korotkikh electromagnithykh impulsov v silitsenovykh volnovodakh [The Dynamics of Ultra-Short Electromagnetic Pulses in Silicene Waveguides]. Pisma v zhurnal tekhnicheskoj fiziki [Technical Physics Letters], 2013, vol. 39 (12), pp. 87-94.

4. Landau L.D., Lifshits E.M. Teoreticheskaya fizika. T. II, Teoriya polya [Theoretical Physics. Vol. 2. Field Theory]. Moscow, Nauka Publ., 1988. 512 p.

5. Yanyushkina N.N., Belonenko M.B., Lebedev N.G. Vliyanie spin-orbitalnogo vzaimodeystviya na dinamiku predelno korotkikh impulsov v grafenovykh sistemakh [The Influence of Spin-Orbit Interaction on the Dynamics of Ultra-Short Pulses in Graphene Systems]. Optika i spectroskopiya [Optics and Spectroscopy], 2012, vol. 112 (3), pp. 498-501.

6. Aufray B., Kara A., Vizzini S., Oughaddou H., Leandri C., Ealet B., Le Lay G. Graphene-Like Silicon Nanoribbons on Ag (110): A Possible Formation of Silicene. Appl. Phys. Lett., 2010, vol. 96, pp. 183102.

7. Ezawa M. Topological Insulator and Helical Zero Mode in Silicone Under Inhomogeneous Electric Field. New J. Phys., 2012, vol. 14, pp. 033003.

8. Ezawa M. Valley-Polarized Metals and Quantum Anomalous Hall Effect in Silicone. Phys. Rev. Lett., 2012, vol. 109, pp. 055502.

9. Lalmi B., Oughaddou H., Enriquez H., Kara A., Vizzini S., Ealet B., Aufray B. Epitaxial Growth of a Silicene Sheet. Appl. Phys. Lett., 2010, vol. 97, pp. 223109.

10. Novoselov K.S., Geim A.K., Morozov S.V., Jiang D., Zhang Y., Dubonos S.V., Grigorieva I.V., Firsov A.A. Electric Field Effect in Atomically Thin Carbon Films. Science, 2004, vol. 306, pp. 666-669.

11. Novoselov K.S., Jiang D., Schedin F., Booth T.J., Khotkevich V.V., Morozov S.V., Geim A.K. TwoDimensional Atomic Crystals. Proc. NatlAcad. Sci. USA, 2005, vol. 102 (no. 30), pp. 10451.

12. Padova P., Quaresima C., Ottaviani C., Sheverdyaeva P., Moras P., Carbone C., Topwal D., Olivieri B., Kara A., Oughaddou H., Aufray B., Lay G. Evidence of Graphene-Like Electronic Signature in Silicone Nanoribbons. Appl. Phys. Lett., 2010, vol. 96, pp. 261905. 


\section{COMPUTER MODELING OF ULTRA-SHORT OPTICAL PULSES INTERACTION} IN SILICENE

\section{Konobeeva Natalia Nikolaevna}

Candidate of Physical and Mathematical Sciences, Associate Professor, Department of Information Systems and Computer Modeling, Volgograd State University yana_nn@inbox.ru, infomod@volsu.ru Prosp. Universitetsky, 100, 400062 Volgograd, Russian Federation

Abstract. There is considerable interest in studying materials with graphene-like structures possessing unique electrical properties, which are promising for micro- and nanoelectronics. One of these materials is silicone - a monolayer of silicon atoms with a hexagonal lattice, which is characterized by a stronger spin-orbit interaction as compared to that in graphene. In addition, it is important that silicon is still the main element in modern electronics.

In this paper we investigate the problem of modeling the ultra-short optical pulses propagation and interaction in silicene. Electrons were considered in long-wave approximation for Hamiltonian in the case of law temperatures. The dependence of pulse form on its amplitude was obtained. The obtained results show that interaction between the ultra-short optical pulses may be useful in light controlling device and can be, for example, the base for an analog comparator of pulse amplitudes.

It should be noted, that when pulse speed increases then the magnitude of the spatial localization of a solitary pulse and the time over which a single pulse "passes" through the other decrease. All this leads to the fact that the effects associated with the nonlinear interaction of pulses does not have time to develop, and the collision is an "elastic" way.

Key words: computer modeling, silicene, ultra-short optical pulses, collision, Maxwell equations. 